\title{
CIRCADIAN RHYTHMS OF ARTERIAL BLOOD PRESSURE IN ISCHEMIC AND HEMORRHAGIC STROKE
}

\author{
Alexander Stoynev \\ Department of Pathophysiology, Sofia Medical University, Bulgaria
}

Circadian variations of arterial blood pressure (ABP) measured by ambulatory BP monitoring demonstrate a "dipper" pattern in healthy individuals of both genders, with a decline in nocturnal ABP. "Nondipper" ABP circadian pattern is considered abnormal because of its association with advanced target organs damage and worse prognosis (1). Patients with secondary hypertension and some patients with primary hypertension have a high risk of stroke, and abnormal circadian $\mathrm{ABP}$ patterns are more prevalent in patients with stroke (70\%-90\% versus $<50 \%$ in nonstroke populations) $(1,2)$.

The mechanisms of altered circadian ABP patterns are not yet fully elucidated, and are considered multifactorial, with an important role of the autonomic nervous system. Acute stroke may alter the autonomic function and thereby affect the circadian pattern of the ABP (2). In patients with acute hemispheric ischemic stroke, increased variability of heart rate and ABP is observed, accompanied by a correlation between the systolic BP and the severity of the autonomic dysfunction, as reported later in this issue (3).

The data about circadian BP patterns and autonomic function in patients with transient ischemic attack or stroke are still scarce. The circadian rhythm of ABP shows significant differences between stroke subtypes in the acute phase, and it is associated with the outcome of the disease (4). In patients with hemorrhagic stroke a higher incidence of abnormal circadian characteristics of ABP - a larger circadian amplitude of systolic BP and a trend to a nondipper pattern are observed, in comparison with ischemic stroke patients (5).

In summary, an abnormal circadian rhythm of ABP is associated with cerebrovascular damage including hemorrhaging, thrombosis, and vascular dementia. Silent cerebrovascular damage is frequently detected in seemingly healthy hypertensive elderly subjects and is also more common among nondippers than in dippers (1). Thus, the ambulatory ABP monitoring provides important data about circadian rhythm of ABP, characterizing autonomic dysfunction in stroke.

\section{REFERENCES}

1. Stoynev, A.G, Ikonomov, O.C., Minkova, N.K., Zacharieva, S.Z., Stoyanovsky, V.G. Circadian rhythms of arterial pressure: basic regulatory mechanisms and clinical value. Acta Physiol Pharmacol Bulg. 1999; 24(3): 43-51.

2. Zhang, W.W., Cadilhac, D.A., Churilov, L., Donnan, G.A., O’Callaghan, C., Dewey, H.M. Does abnormal circadian blood pressure pattern really matter in patients with transient ischemic attack or minor stroke? Stroke. 2014; 45: 865-867.

3. Georgieva-Hristova, D., Andonova, S., Kalevska, E., Drenski, T., Boyadzhieva, M. Arterial blood pressure and heart rate response in patients with acute ischemic stroke correlation with the severity of cardiovascular autonomic dysfunction. Scripta Sci Med. 2016; 48(2): 12-17.

4. Zis, P., Vemmos, K., Spengos, K., Manios, E., Zis, V., Dimopoulos, M.A., Zakopoulos, N. Ambulatory blood pressure monitoring in acute stroke: pathophysiology of the time rate of blood pressure variation and association with the 1-year outcome. Blood Press Monit. 2013; 18(2): 94-100.

5. Jiang, Z., Li, W., Liu, Y., Wang, Y., Wang, Z., Xiao, J., Guo, H., Cornélissen, G., Halberg, F. Circadian blood pressure and heart rate characteristics in haemorrhagic vs ischaemic stroke in Chinese people. J Hum Hypertens. 2010. 24(3): 165-174. 